\title{
Control enfocado de Aedes aegypti en localidades de alto riesgo de transmisión de dengue en Morelos, México
}

\author{
Alejandro Villegas-Trejo, M en C,(I) Azael Che-Mendoza, M en C,(I) Mariana González-Fernández, M en C,(I) \\ Guillermo Guillermo-May, Biol,(2) Hugo González-Bejarano, MSP, M en Admon, (1) Felipe Dzul-Manzanilla, M en C, (3) \\ Armando Ulloa-García, PhD (4) Rogelio Danis-Lozano, PhD, (4) Pablo Manrique-Saide, PhD. (2)
}

\begin{abstract}
Villegas-Trejo A, Che-Mendoza A, González-Fernández M, Guillermo-May G, González-Bejarano H, Dzul-Manzanilla F, Ulloa-García A, Danis-Lozano R, Manrique-Saide P. Control enfocado de Aedes aegypti en localidades de alto riesgo de transmisión de dengue en Morelos, México. Salud Publica Mex 20II;53:14I-15I.
\end{abstract}

\section{Resumen}

Objetivo. Determinar la importancia relativa de los tipos de criadero de Aedes aegypti para proponer intervenciones de control enfocadas en Morelos. Material y métodos. Se realizaron muestreos transversales con colecta de pupas en Cuautla, Jojutla y Tlaquiltenango en temporada de sequía (I 7 I3 casas) y lluvias (I 677) del año 2008. La importancia relativa de cada tipo de criadero se determinó por su contribución (\%) a la producción pupal total por localidad. Resultados. En Cuautla, la mayoría de pupas se recolectaron en temporada de sequía de tanques/pilas (48.5\%), tambos y botes/cubetas ( $15 \% \mathrm{c} / \mathrm{u})$; en lluvias, los diversos chicos (21.3\%), botes/cubetas (19.3\%) y macetas/macetones (12.9\%) fueron más productivos. En Jojutla y Tlaquiltenango, $97 \%$ de las pupas se colectaron de macetas/macetones en secas; durante las lluvias la mayoría de pupas se recolectaron de diversos chi$\cos (26.3 \%)$, trastes de cocina/lavado (13.9\%), botes/cubetas (I2.9\%) y macetas/macetones (I2.7\%), respectivamente. Conclusión. La prevención y control del vector del dengue deben basarse en este tipo de evidencias para focalizar las acciones sobre los criaderos más productivos.

Palabras clave: dengue; Aedes aegypti; control; productividad; Morelos; México
Villegas-Trejo A, Che-Mendoza A, González-Fernández M, Guillermo-May G, González-Bejarano H, Dzul-Manzanilla F, Ulloa-García A, Danis-Lozano R, Manrique-Saide P. Targeted treatment of Aedes aegypti at localities with high risk for dengue transmission, Morelos, Mexico. Salud Publica Mex 20 I; 53:14I-15I.

\begin{abstract}
Objective. To determine the relative importance of Aedes aegyti breeding sites for potential targeted dengue control interventions in Morelos. Material and Methods. Crosssectional entomological surveys were conducted and collection of Ae. aegypti pupae was taken from all water-holding containers in Cuautla, Jojutla and Tlaquiltenango during dry (I 7/3 households) and rainy (I 677) seasons in 2008. Relative importance of different types of breeding sites was determined by the contribution (\%) to total pupae production within each locality. Results. In Cuautla most pupae during the dry season were found in wash basins $(48.5 \%)$, tanks and buckets/pots ( $15 \%$ each); during the rainy season, diverse small items (21.3\%), buckets/pots (19.3\%) and plant pots (12.9\%) were more productive. In Jojutla and Tlaquiltenango, $97 \%$ of all pupae was found in plant pots during the dry season; during the rainy season diverse small items (26.3\%), washing/cooking utensils (13.9\%), buckets/pots (12.9\%) and plant pots (12.7\%) were significant. Conclusion. Prevention and control of the dengue vector should be based on this kind of evidence to target the most productive breeding-sites.
\end{abstract}

Key words: dengue; Aedes aegypti; control; productivity; Morelos; Mexico

(I) Servicios de Salud de Morelos. Cuernavaca, Morelos, México.

(2) Departamento de Zoología, CA Bio-Ecología Animal, Campus de Ciencias Biológicas y Agropecuarias (CCBA), Universidad Autónoma de Yucatán (UADY). Mérida, Yucatán, México.

(3) Servicios Estatales de Salud de Guerrero. Chilpancingo, Guerrero, México.

(4) Centro Regional en Investigación en Salud Publica, Instituto Nacional de Salud Pública. Tapachula, Chiapas, México.

Fecha de recibido: 28 de enero de 2010 - Fecha de aceptado: 12 de enero de 2011

Solicitud de sobretiros: Dr. Pablo Manrique Saide. Departamento de Zoología, Campus de Ciencias Biológicas y Agropecuarias,

Universidad Autónoma de Yucatán.Apartado Postal 4-1 16, Itzimná. 97100 Mérida Yucatán, México.

Correo electrónico:msaide@uady.mx 
E Programa de Prevención y Control del Dengue de todos los estados de México, incluido Morelos, está basado en la reducción de criaderos de Aedes aegypti a través del control físico (eliminación, remoción y protección), químico (aplicación de larvicida temefos granulado, v.g. abate con visitas casa por casa y rociados espaciales para la eliminación de adultos) y complementado con campañas de descacharrización anunciadas en medios masivos de comunicación. Asimismo, se promueven acciones de limpieza domiciliaria por la comunidad denominadas "patio limpio". ${ }^{1}$

El estado de Morelos registró el brote de dengue más importante de su historia durante 2008 con 5953 casos acumulados de fiebre por dengue (FD) y 2165 casos de fiebre hemorrágica por dengue (FHD), con una tasa de incidencia de 489 por 100000 habitantes y una tasa de letalidad de 0.7 por 100 casos. Ante la experiencia de 2008, el programa estatal de control de dengue replanteó parte de sus estrategias con base en nuevos lineamientos para el control de vectores: ${ }^{2}$ el manejo integrado del dengue, de acuerdo con el riesgo entomológico, y la estratificación dinámica de casos probables (riesgo epidemiológico) con acciones inmediatas e integrales. Con respecto a las intervenciones entomológicas, se seleccionaron métodos que inciden en las poblaciones del vector a nivel local, lo que marca la pauta para el control enfocado y que potencialmente reduzca a niveles significativos la transmisión de la enfermedad y la morbilidad; los métodos de control seleccionados emplearán herramientas de comprobado costo-efectividad, pudiendo incluir una combinación de intervenciones usadas en sinergia.

La reducción de Ae. aegypti con participación comunitaria y la aplicación de larvicidas (dentro de una gama de productos aprobados por la Secretaría de Salud) son intervenciones de bajo impacto ambiental y recomendadas en los nuevos lineamientos de esta dependencia. Sin embargo, su efecto sostenido en las poblaciones del mosquito vector es limitado si se aplica de forma generalizada y sin considerar que la importancia de los tipos de criaderos de Ae. aegypti no es la misma en cuanto a su productividad (aquellos responsables de producir la mayor proporción de pupas y de mosquitos adultos) $)^{3-5}$ ni las variaciones geográficas (entre localidades y al interior de ellas) y temporales (estacionales).

Por esta razón, se recomienda que la selección de los criaderos que serán controlados en las campañas del dengue emplee evidencia local y consistente de su productividad pupal. La estrategia ha sido evaluada con éxito en diferentes países para identificar los tipos de criaderos más productivos, llamados contenedores clave, de pupas de Ae. aegypti. ${ }^{6-12}$ En México, se realizaron dos estudios recientes en Chiapas y Yucatán, donde se reportó que los tanques y las cubetas, en uno y otro estado, respectivamente, fueron los criaderos más productivos, cuyo control no se consigue en su totalidad durante las campañas actuales basadas en la descacharrización y con el control químico. ${ }^{13,14}$

El presente estudio demuestra la importancia relativa de los diferentes tipos de criaderos de Ae. aegypti con base en su productividad pupal durante las temporadas de secas y lluvias, en tres localidades urbanas de Morelos de alto riesgo de transmisión de dengue. Se discuten implicaciones prácticas de los resultados y se presenta un análisis básico de los costos asociados a su implementación, y un rango de intervenciones y herramientas posibles con la finalidad de proponer estrategias de control enfocadas a estos criaderos.

\section{Material y métodos}

Área de estudio. El estudio se realizó en tres localidades del estado de Morelos: Jojutla (N18³6'53", O99¹0'49"), con una altitud de 890 msnm y 24901 habitantes; Tlaquiltenango (N18 37'44", $\left.\mathrm{O}^{\circ} 9^{\circ} 09^{\prime} 37^{\prime \prime}\right)$, con una altitud de 920 msnm y 27223 habitantes; y Cuautla (N18 $48^{\prime} 44^{\prime \prime}$, O98 57'21'), con una altitud de 1300 msnm y 215950 habitantes (figura 1). La distancia entre las localidades de estudio y la capital del estado es de $34 \mathrm{~km}$ y entre Cuautla, Jojutla y Tlaquiltenango de $32 \mathrm{~km}$. Estas localidades forman parte de siete ciudades de alto riesgo de transmisión de dengue en las zonas urbanas del sur y del este de Morelos. Durante 2006, Cuautla presentó el brote más grande del estado con 833 casos de dengue y una tasa de incidencia de 498 por 100000 habitantes; por su parte, Jojutla y Tlaquiltenango reportaron 230 y 206 casos con tasas de incidencia de 458 y 705 por 100000 habitantes en una y otra localidad, respectivamente, y la circulación del serotipo DENV-3. Dos años después, Cuautla registró 836 casos de dengue y una tasa de 500 por 100000 habitantes, mientras que Jojutla y Tlaquiltenango registraron 533 y 138 casos y tasas de 1061 y 472 por 100000 habitantes, con la circulación del serotipo DENV-1. ${ }^{15,16}$

El clima de las tres localidades es de tipo Aw"0(w) (i')g, cálido subhúmedo, ${ }^{17}$ que se caracteriza por dos estaciones: una seca de noviembre a mayo y otra lluviosa de junio a octubre. Durante los meses de febrero-marzo, en Jojutla y Tlaquiltenango la temperatura promedio fue de $24^{\circ} \mathrm{C}$ con una precipitación pluvial de $1.1 \mathrm{~mm}$, y durante julio y agosto se registró una temperatura y una precipitación pluvial promedio de $25^{\circ} \mathrm{C}$ y $38 \mathrm{~mm}$, respectivamente. En Cuautla, la temperatura y precipitación pluvial promedio durante la temporada de secas fueron de $22^{\circ} \mathrm{C}$ y $2.6 \mathrm{~mm}$, mientras que en la temporada 


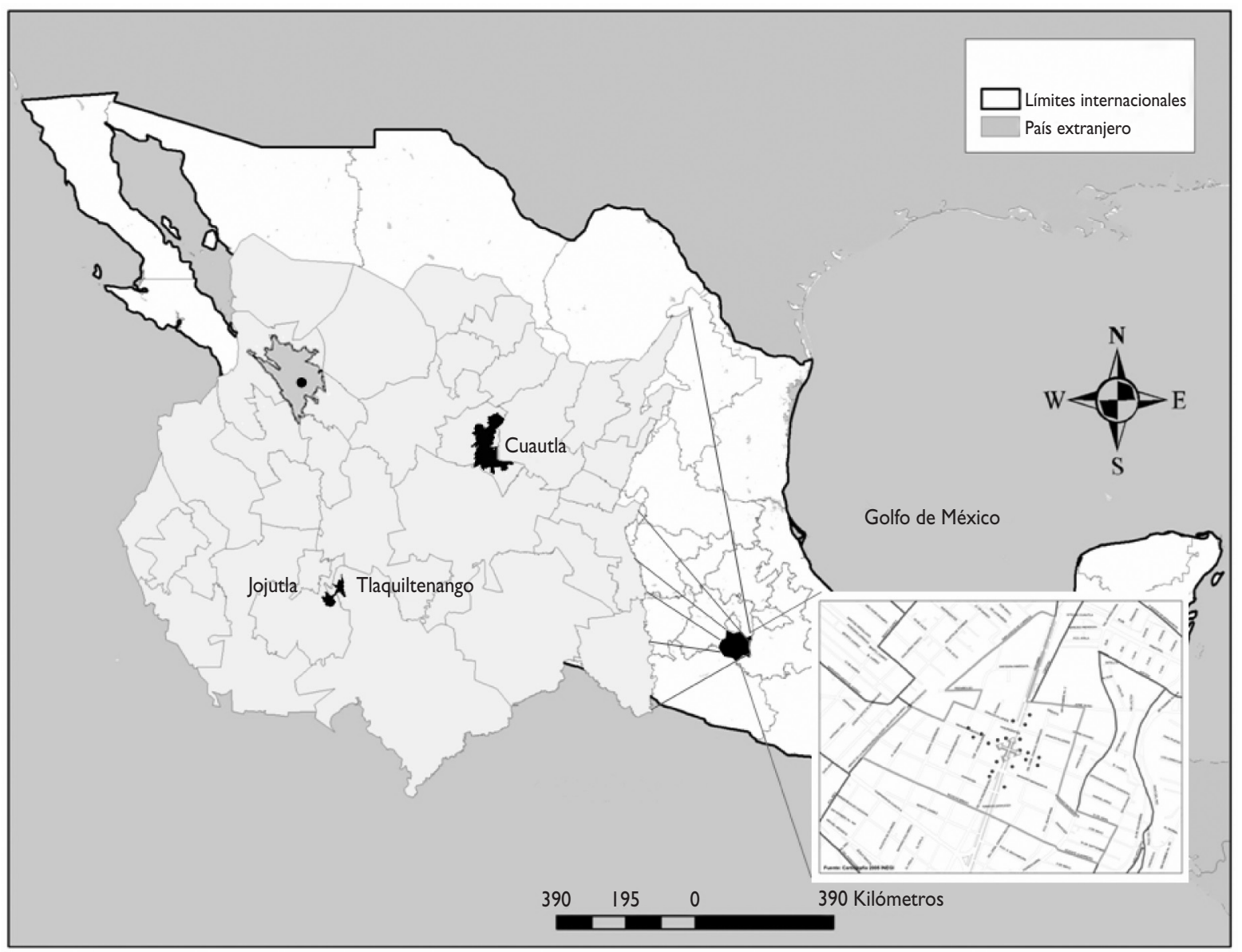

Figura I. Localidades de estudio. Un punto señala la capital del estado, Cuernavaca, Morelos. Representación ESQUEMÁTICA DEL MUESTREO DE UN AGEB. LAS CASAS A INSPECCIONAR SE REPRESENTAN MEDIANTE PUNTOS

de lluvias la temperatura promedio fue de $22.8^{\circ} \mathrm{C}$ con una precipitación pluvial de $40.3 \mathrm{~mm} .{ }^{18}$

Diseño del estudio y tamaño de muestra. Se realizaron dos muestreos transversales, uno de ellos en la temporada de secas (febrero a marzo) y otro durante la temporada de lluvias (julio-agosto) de 2008. El muestreo se realizó por conglomerados al utilizar el Área Geoestadística Básica (AGEB) como unidad. El 100\% de los AGEB fueron muestreados en cada localidad y en cada uno de ellos se revisaron 20 casas cada temporada; en Cuautla se muestrearon $73 \mathrm{AGEB}$, con un promedio de 821 casas por AGEB (en lluvias se redujo a 69 AGEB debido a ajustes derivados de la fusión de las mismas en la periferia de la localidad, que tenían menos de 20 casas) y en Jojutla y Tlaquiltenango 15 AGEB, con un promedio de 965 casas por cada una de ellas. Un total de 1713 y 1677 viviendas fueron muestreadas en temporada de secas y lluvias, respectivamente. El método de selección de las casas fue el empleado por Manrique-Saide y colaboradores. ${ }^{14} \mathrm{Se}$ ubicó el centro del AGEB y desde allí se seleccionaron cinco casas, alternando una en cada lado de la calle y dejando entre 3 a 5 casas por cada casa inspeccionada, hasta completar 20 (5/punto cardinal) (figura 1$)$. Se explicó a los moradores el objetivo del estudio y posteriormente se pidió su autorización para ingresar al domicilio a realizar las colectas entomológicas.

Colectas entomológicas. Se realizó la búsqueda activa de larvas y pupas de mosquitos en todos los recipientes con potencial para ser criaderos de mosquitos de acuerdo con las recomendaciones de Focks y la Organización Panamericana de la Salud (OPS). 3,19 Cada uno fue considerado positivo cuando al menos una larva de cualquier estadio o pupa estuvo presente y se clasificaron de acuerdo con su localización 
dentro o fuera de las casas; se registraron los datos de capacidad, origen natural o artificial, material de manufactura y si la familia lo consideraba desechable o útil. Las colectas se llevaron a cabo entre las 7:00 y las 15:00 horas por equipos conformados por dos personas especializadas en actividades de entomología del programa de dengue del estado (con un estimado total de 20 casas diarias por equipo) y con el uso de un formato previamente validado. Todos los especímenes (larvas y pupas) recolectados se preservaron en viales con alcohol para su transporte al laboratorio donde se realizó la identificación taxonómica usando las claves de Darsie, Darsie y Ward e Ibáñez-Bernal-Martínez $\mathrm{C}^{20-22}$ y en el sitio de internet de la unidad de Biosistemática Walter Reed (WRBU). ${ }^{23}$ Las pupas muertas o sus exuvias encontradas al momento de revisar criaderos también fueron identificadas. Parte de las muestras fueron enviadas al Laboratorio Estatal de Salud Pública (LESP) de Guerrero para su confirmación y / o determinación taxonómica a nivel de especie. Una muestra de los especímenes colectados se encuentran preservados en viales con alcohol a $70 \%$ y etiquetados con datos del AGEB, número de casa, tipo de criadero, material de manufactura, capacidad y volumen de agua, y forman parte de una Colección Regional de Artrópodos de Importancia Médica del estado de Morelos, con muestras de referencia (voucher) a la Colección Entomológica Regional de la Universidad Autónoma de Yucatán y en el Área de Parasitología y Entomología del Laboratorio Estatal de Salud Pública de los Servicios Estatales de Salud de Guerrero.

Análisis de datos. Se compararon las abundancias de inmaduros, particularmente su contribución porcentual de pupas de Ae. aegypti entre los tipos de criaderos encontrados en las viviendas. Las variables consideradas para el análisis incluyeron: el número total de recipientes por categoría (n), recipientes con agua (RA), número absoluto de pupas, media aritmética y desviación estandar del total de pupas por recipiente positivo y su valor máximo, recipientes con agua positivos a larvas y/o pupas de Ae. aegypti (RP) que se interpreta como "índice de recipiente" (IR), recipientes positivos a pupas de Ae. aegypti (RPPU) y la contribución porcentual por recipiente del total de pupas (\% de productividad de un recipiente) definida como el número de pupas en el total de recipientes de una categoría, dividido entre el número total de pupas en todos los recipientes. ${ }^{6,24,25} \mathrm{La}$ significancia de la productividad pupal entre los contenedores por localidad y por temporada se comparó mediante la prueba de $\chi^{2}$ El protocolo fue revisado por la Comisión de Ética del Instituto Nacional de Salud Pública.

\section{Resultados}

\section{Proporción de especies en recipientes artificiales}

Un total de 1303 recipientes ( $4.9 \%$ de los RA) fueron positivos a inmaduros de Aedes spp. El 60.8\% ( $\mathrm{n}=792$, $2.9 \%$ de los RA) fueron positivos a pupas del género Aedes: $37.5 \%$ (489, 1.8\% RA) fue positivo a pupas de $A e$. aegypti, $15.8 \%(206,0.8 \%$ RA) a Ae. epactius y $7.4 \%$ (97, $0.4 \%$ RA) positivo a pupas de ambas especies.

\section{Frecuencia relativa de recipientes artificiales en las dos temporadas del año}

\section{Temporada de secas}

Se revisaron 1713 viviendas de las tres localidades, con 97142 recipientes caracterizados y clasificados en 22 categorías generales (cuadro I): macetas y macetones (31.7\%); botellas $(31.5 \%)$; botes y cubetas $(10.8 \%)$; diversos chicos (6.7\%); trastes de cocina y lavado (4.7\%); diversos grandes (2.5\%); aparatos inservibles $(1.9 \%)$; tambos (1.6\%); tinacos (1.4\%); bebederos (1.3\%); tanques $(1.2 \%)$; latas (1.1\%); llantas, naturales, cisternas, floreros, piletas, pozos, artículos de baño, albercas, fuentes y otros, (cada uno representa menos de 1\%). El 11.2\% del total de recipientes se encontraron con agua y $96 \%$ se localizaron en el peridomicilio, tanto en Cuautla como en Jojutla y Tlaquiltenango.

Cuautla. De 79439 recipientes revisados, $11.9 \%$ se encontró con agua (con un promedio de $7 \mathrm{RA} / \mathrm{CAS}$ ). Las botellas, botes y cubetas fueron los tipos de recipiente con agua más abundantes (19.7 y 17.6\% de todos los RA respectivamente). La mayor prevalencia de infestación por Ae. aegypti (IR) se registró en fuentes $(4.3 \%$ ) y tanques y pilas (2\%). Sin embargo, los tipos de recipiente más productivos fueron los tanques / pilas (con $48.5 \%$ de todas las pupas recolectadas), los cuales, junto con tambos, botes y cubetas, acumularon $78.5 \%$ de la producción total de pupas (cuadro I). Únicamente los tanques fueron mejores productores de pupas que los botes y cubetas $\left(\chi^{2}=8.96 P=0.003\right)$. Entre tambos, botes y cubetas, no se encontró diferencia significativa $\left(\chi^{2}=0.16 P=0.69\right)$.

Jojutla/Tlaquiltenango: De 17703 recipientes revisados, $7.6 \%$ se encontró con agua (con un promedio de $5 \mathrm{RA} /$ CAS). Los tanques/ pilas, botes y cubetas fueron los RA más abundantes (18.5 y 15.1\% de todos los RA, respectivamente). La mayor prevalencia de infestación por 


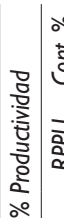

蓆

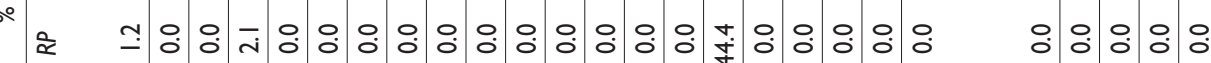

$\therefore$

$\stackrel{\dot{x}}{\Sigma}$

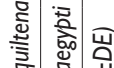

这安

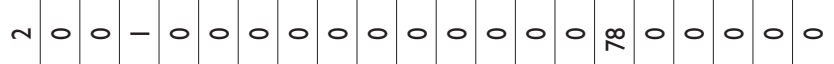

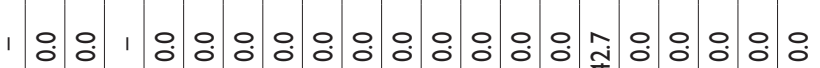

00000

$\therefore$ 웅영웅웅

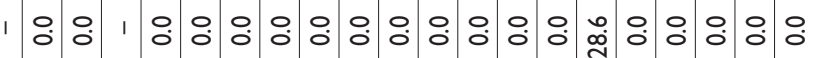

웅영웅웅웅

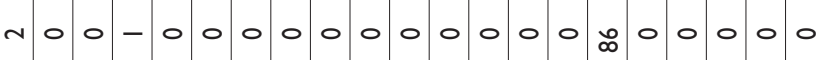

$\circ 00000$

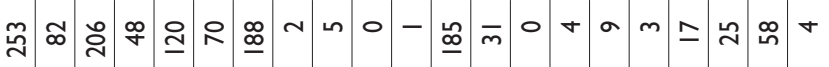

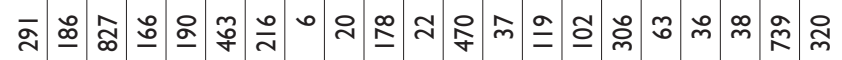

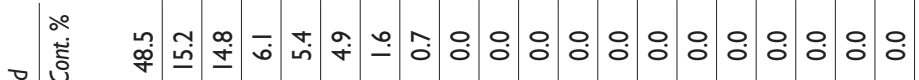

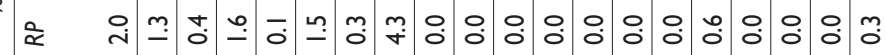

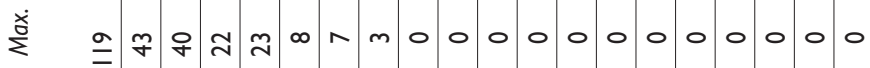

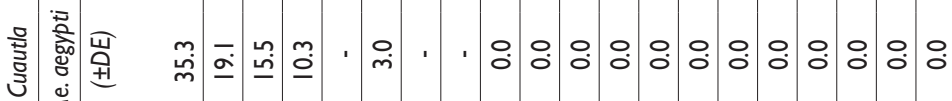

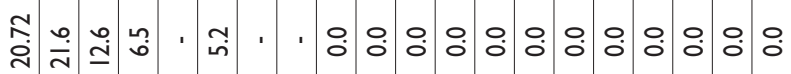

aิ่

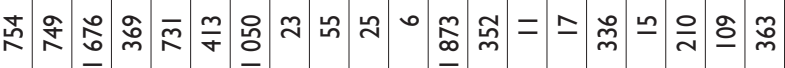

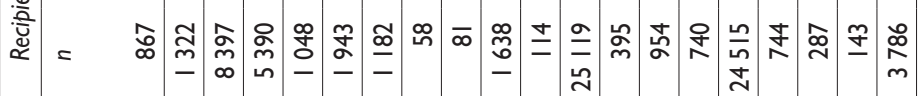
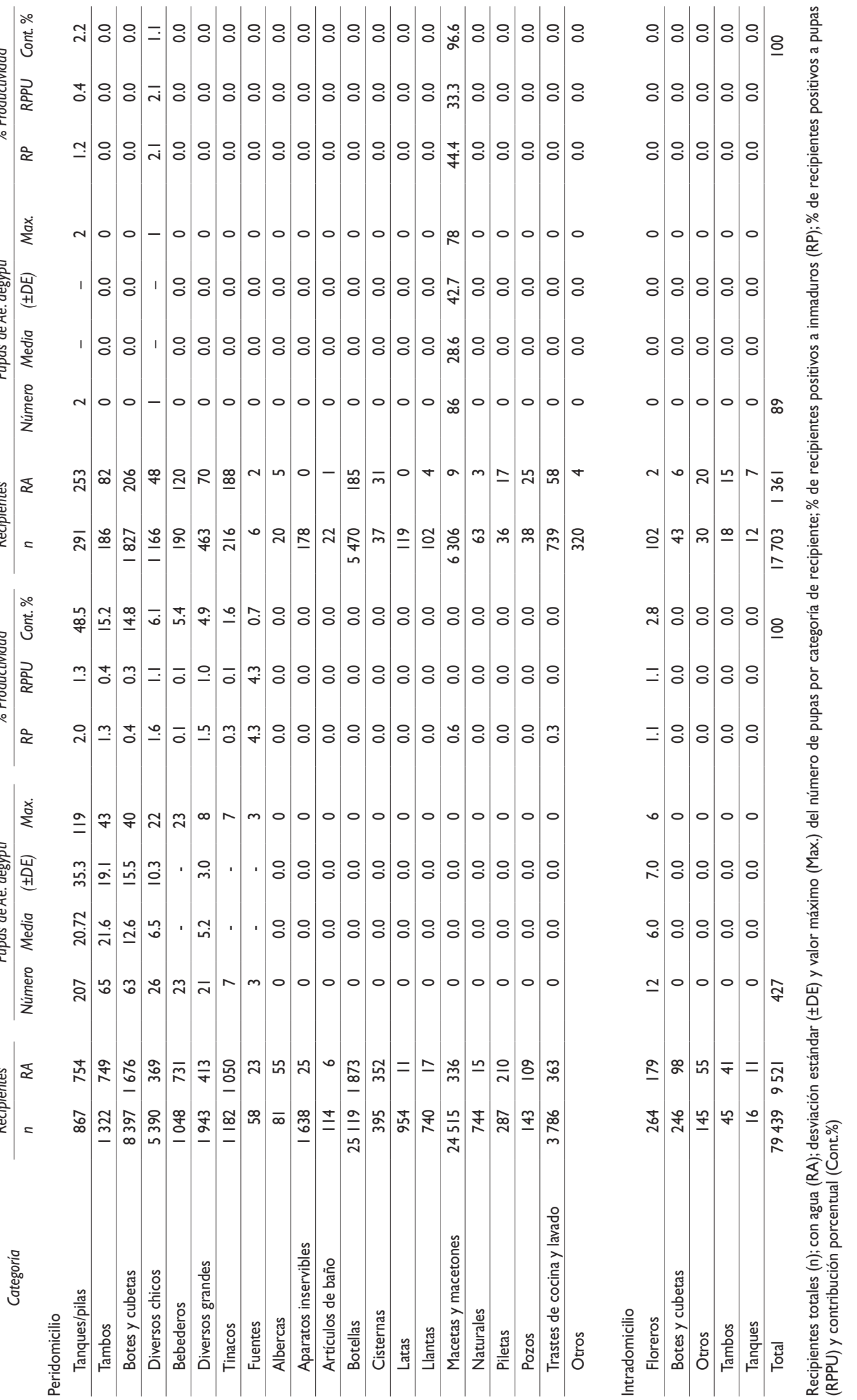
Ae. aegypti (IR) se observó en las macetas y macetones $(\mathrm{IR}=44.4 \%)$. Estos últimos recipientes junto con los diversos chicos y los tanques/pilas fueron los únicos positivos a pupas de Ae. aegypti. Sin embargo, $97 \%$ de todas las pupas recolectadas se encontraron en macetas y macetones (cuadro I).

\section{Temporada de lluvias}

Se revisaron 1677 viviendas de las tres localidades, con 113417 recipientes de 22 categorías: macetas y macetones $(30.4 \%)$; botellas $(28.4 \%)$; botes y cubetas (9.8\%); trastes de cocina y lavado ( $7.8 \%)$; diversos chicos $(6.6 \%)$; latas $(5.7 \%)$; diversos grandes $(2.8 \%)$ aparatos inservibles $(1.3 \%)$; tambos (1.3\%); bebederos $(1.2 \%)$; tinacos $(1.1 \%)$; tanques $(1.0 \%)$; llantas, piletas, cisternas, artículos de baño, floreros, naturales, pozos, albercas y fuentes y otros (cada uno menos del 1\%). El 13.1\% del total de recipientes se encontró con agua y $97 \%$ se localizó en el peridomicilio en las tres localidades.

Cuautla. De 91026 recipientes revisados, 14.3\% se encontró con agua (con un promedio de 7 RA/CAS). Los botes y cubetas, así como, diversos chicos fueron los RA más abundantes (20.2 y 12.4\% de todos los RA, respectivamente). La mayor prevalencia de infestación por Ae. aegypti se observó en llantas (19\%) y fuentes (19\%). Sin embargo, los diversos chicos $(21.3 \%)$, botes y cubetas $(19.3 \%$ ) y macetas y macetones $(12.9 \%)$ fueron responsables de producir $53.5 \%$ del total de las pupas recolectadas durante las lluvias (cuadro II). Los diversos chicos fueron mejores productores de pupas que los botes y cubetas $\left(\chi^{2}=20.66 P=0.001\right)$, pero no que las macetas y macetones $\left(\chi^{2}=0.65 P=0.42\right)$. Por su parte, los botes y cubetas fueron mejores productores de pupas que las macetas y macetones $\left(\chi^{2}=8.31 P=0.003\right)$.

Jojutla/Tlaquiltenango: De 22391 recipientes revisados, 8.2\% se encontró con agua, (con un promedio de 6.1 RA/CAS). Los botes y cubetas, tanques/pilas y los bebederos fueron los RA más abundantes $(15.5,13.3$ y $10.6 \%$ de todos los RA, respectivamente). La mayor prevalencia de Ae. aegypti se observó en latas $(\mathrm{IR}=37.5 \%)$, artículos de baño $(30.8 \%)$, diversos chicos $(27.2 \%)$ y aparatos inservibles (25\%). Sin embargo, se encontró que los tipos de recipiente más productivos del total de pupas recolectadas fueron: diversos chicos $(26.3 \%)$, trastes de cocina y lavado (13.9\%), botes y cubetas $(12.9 \%)$ y macetas y macetones $(12.7 \%)$. Estas cuatro categorías de criaderos produjeron $65.8 \%$ del total de pupas (cuadro II). Sólo los botes y cubetas fueron mejores productores de pupas que los trastes de cocina y lavado $\left(\chi^{2}=7.02 P=008\right)$.

\section{Discusión}

Los resultados presentados son similares a los reportados por diversos autores. ${ }^{5,6,11,13,14,24,26}$ Se reporta que un número reducido de recipientes $(2.2 \%)$ concentró la mayor cantidad de pupas de Ae. aegypti en las tres localidades de estudio (figura 2). Los indicadores de éxito de la mayoría de los programas de control de dengue se enfocan inicialmente en la reducción de la densidad de estadios inmaduros de Ae. aegypti $i^{27}$ y posteriormente a la disminución de adultos. Un control eficaz dirigido sobre los criaderos más productivos de pupas podría tener mayor impacto sobre las poblaciones del vector.

La localización, el uso y la temporalidad parecen ser factores determinantes de la producción pupal de los recipientes. Por ejemplo, del total de criaderos con pupas, 93\% estuvo localizado en los patios de las casas; así que dirigir y centrar los esfuerzos hacia estos recipientes puede tener importantes ventajas logísticas en el control y la vigilancia entomológica del dengue. Si bien la identidad de los criaderos más productivos difiere entre localidades, la característica que tienen en común es que son mantenidos con agua por los habitantes de las casas en la época de secas, en especial en tanques y pilas en Cuautla y macetas y macetones en Jojutla y Tlaquiltenango. Con la llegada de las lluvias aumenta la diversidad y abundancia de los tipos de criaderos disponibles: en Cuautla los diversos chicos (32\% considerados desechables) fueron los más productivos junto con los botes y cubetas, macetas y macetones y los trastes de cocina y lavado; mientras que en Jojutla y Tlaquiltenango los diversos chicos fueron el recipiente más productivo junto con los trastes de cocina y lavado, botes/cubetas y macetas/macetones.

Los tanques y tambos son considerados como necesarios, por lo tanto, es imposible eliminarlos en las campañas de remoción de criaderos. Su existencia está asociada con la deficiencia del suministro de agua potable ${ }^{28}$ (principalmente en Cuautla, donde se registró un tanque y un tambo por cada dos casas en promedio) lo que es una evidencia de los patrones culturales sobre el uso y manejo inapropiado de los recipientes que almacenan agua. La importancia de los tanques en México ha sido reportada anteriormente por Arredondo-Jiménez y Valdez-Delgado ${ }^{13}$ en Chiapas, en donde 4 de cada 5 pupas fueron encontradas en tanques. Los tambos han sido reportados como los recipientes productivos más importantes en otros países como Nicaragua, ${ }^{8}$ Venezuela, ${ }^{9}$ y Colombia, ${ }^{11}$ lo que genera más de $30 \%$ del total de pupas en esas ciudades. 


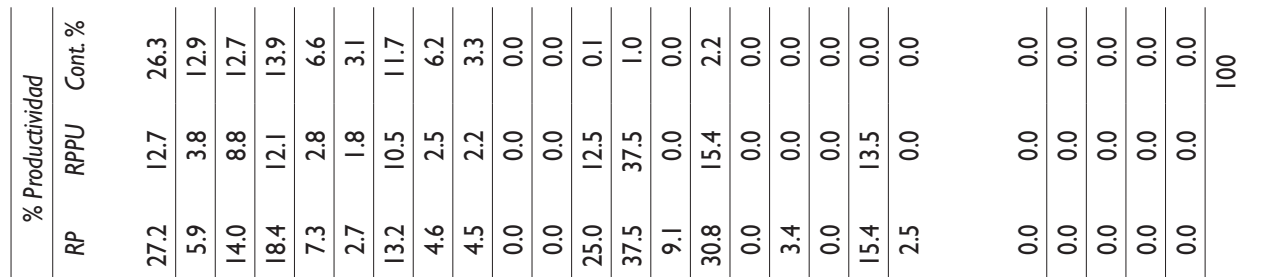

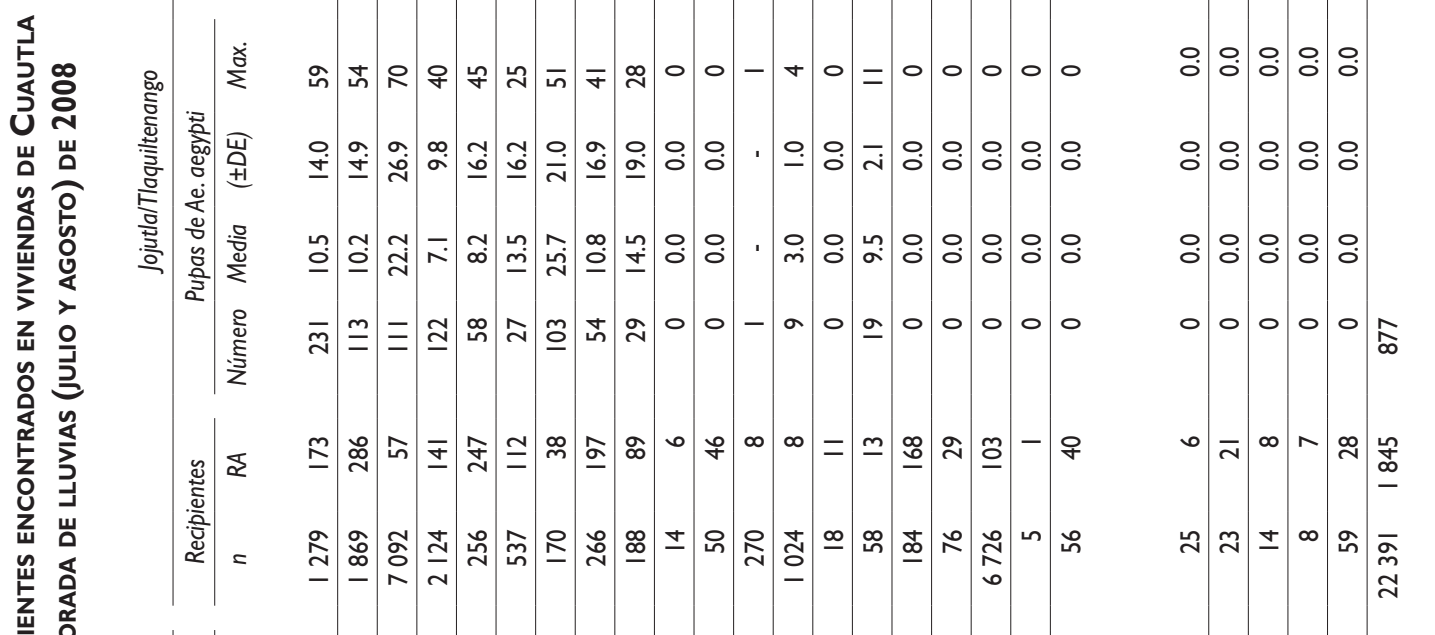

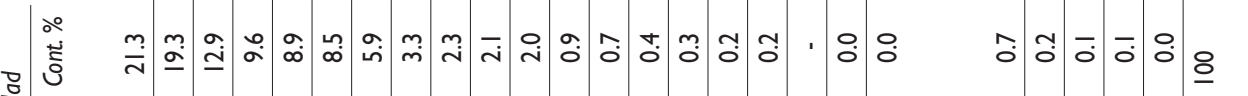

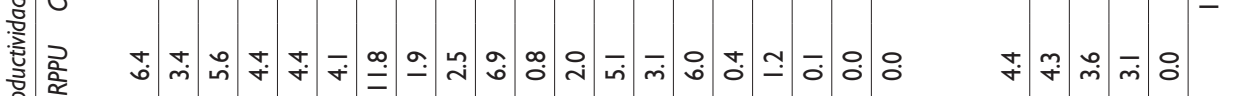

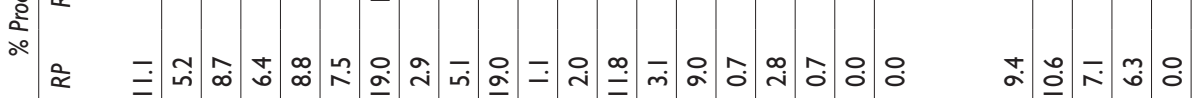

岁号

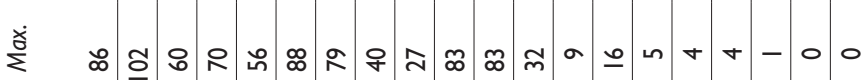

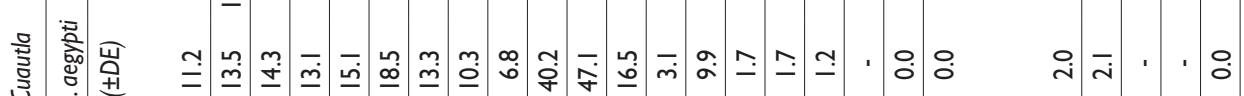

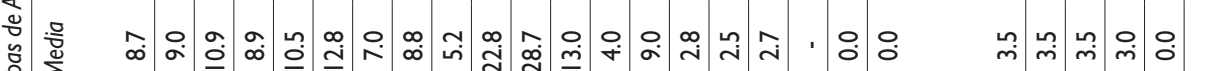

$\frac{2}{2} \sum$

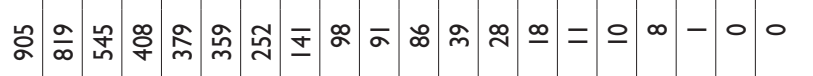

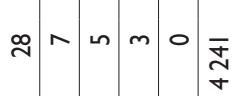

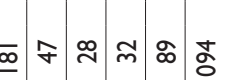

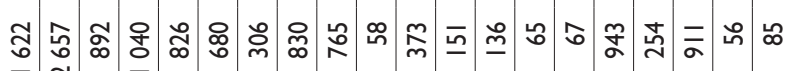

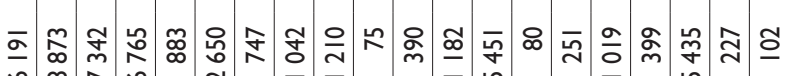

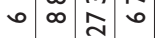
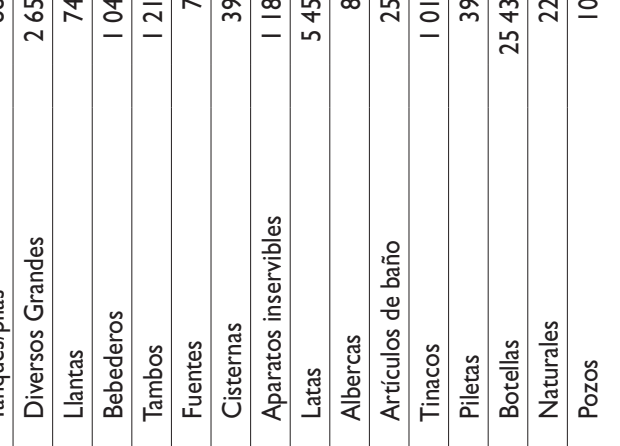

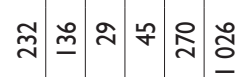

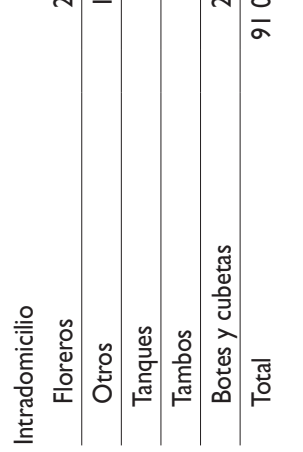

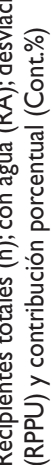




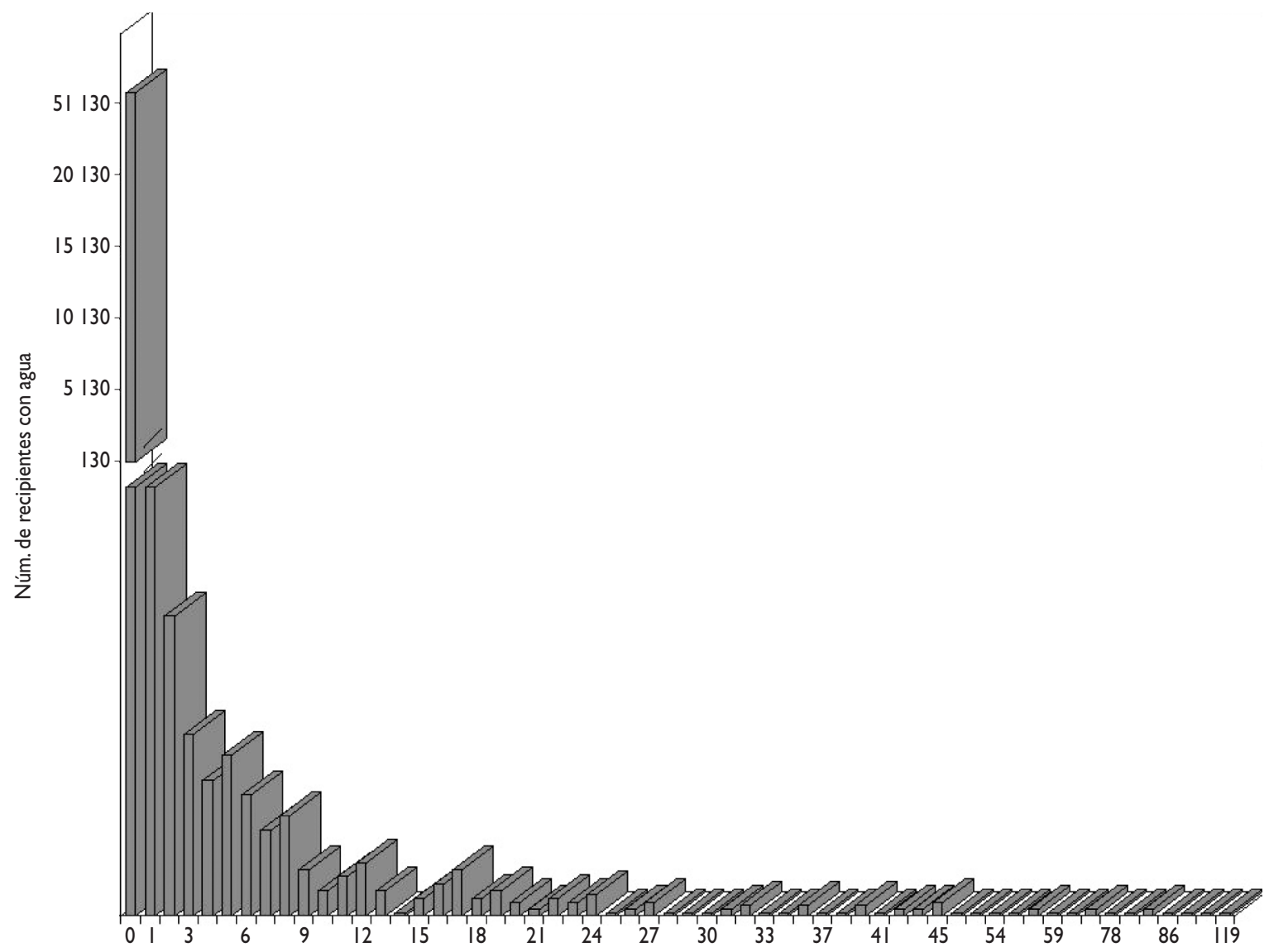

Figura 2. Distribución de frecuencias para el número de pupas ( $\mathbf{N}=5$ 634) De AE. AeGypti enCONTRADAs EN CONTENEDORES CON AGUA ( $\mathrm{N}=25$ 82I). LOS DATOS MOSTRADOS SON UNA COMBINACIÓN DE LOS RESULTADOS DE LOS MUESTREOS EN SECAS $Y$ lluvias de 2008 en las localidades de Cuautla, Jojutla y Tlaquiltenango

En este estudio se considera que parte de la variación temporal en la productividad pupal de los tanques fue resultado de las acciones propias de control del programa de dengue, a pesar de que el promedio de tanques con agua por casa en Jojutla y Tlaquiltenango (3.5 por cada 4 casas) fue mayor que en Cuautla (2 por cada 4 casas). En Cuautla, el porcentaje de tanques con agua desinfectados pasó de $0.13 \%$ en secas a $44.4 \%$ en lluvias, y en Jojutla y Tlaquiltenango de 39 a $72 \%$ en secas y lluvias, respectivamente (confirmado por nuestros muestreos). Particularmente en Jojutla y Tlaquiltenango se observó la presencia de peces (Oreochromis spp) en $17.8 \%$ de los tanques con agua.

La importancia de las cubetas en la productividad pupal también ha sido demostrada previamente en México. En Mérida, Yucatán ${ }^{14}$ se reportó que la presencia de las cubetas de plástico (5-10 litros) ubicadas en los patios fue consistente, por lo que se demostró que este tipo de criadero fue el más abundante y responsable de producir 34\% del total de pupas. Por su parte, las macetas y macetones (generalmente utensilios de diversa manufactura con la característica de contener tierra y plantas no acuáticas) fueron el tipo de recipiente más importante en cuanto a la producción de pupas en Jojutla y Tlaquiltenango.

Los tanques, tambos, cubetas y macetas son los tipos de recipientes sobre los que la población no tiene el cuidado y la prevención para que no se conviertan en criaderos de mosquitos. Los tanques y tambos son normalmente controlados con la aplicación de larvicidas y a la fecha es la alternativa más confiable para el control larvario de Ae. aegypti, a pesar de los costos que genera. Sin embargo, hay que tomar en consideración la aceptación o rechazo de la gente para que el abate en formulación granular permanezca dentro de sus tanques y otros recipientes. Indudablemente, el programa de 
control de dengue en Morelos deberá explorar nuevas alternativas para el control de tanques y tambos con mayor participación comunitaria en estudios futuros, sin dejar de considerar las medidas de control físico como por ejemplo el uso de tapaderas de plástico (tapa tambos). ${ }^{29}$

Resultó evidente la importancia que tienen los botes y cubetas en Cuautla, al ser el tipo de contenedor cuya productividad pupal se mantuvo constante tanto en secas (18\% RA y 14.8\% PU) como en lluvias (20\% RA y 19.3\% PU). Si bien la condición de un contenedor como productivo depende de factores físicos-biológicos (precipitación pluvial, temperatura, humedad, localización, presencia de materia orgánica, disponibilidad, material del contenedor, exposición al sol, etc.), de la conducta de ovipostura del vector (asociado a la preferencia hacia ciertas condiciones microclimáticas que favorecen su desarrollo durante su fase acuática), y de los conocimientos, actitudes y prácticas sobre el uso y manejo que la población humana le da a los contenedores que almacenan agua, su estudio es de interés preponderante para el control de los mismos. ${ }^{30,31}$ Nuestro análisis tiene restricciones en cuanto a la aportación de datos sobre las características físicas-biológicas de los contenedores de agua, aspecto que está asociado a la productividad pupal; i. e. contenedores de gran volumen y protegidos de la luz del sol son más productivos; ${ }^{32,33}$ por lo que resulta importante incluir estas mediciones en posteriores estudios y enriquecer la evidencia sobre la implicación del microhábitat en la formación de criaderos.

El tratamiento preventivo o de control para las cubetas y macetas/macetones (y quizá de tanques y tambos en el corto plazo), deberá estar enfocado al desarrollo de programas educativos que consideren la promoción de la salud y la comunicación social, así como la evaluación de la adopción de las medidas preventivas por parte de la población. ${ }^{34}$ El costo calculado de un programa educativo para una localidad con densidad poblacional de ocho mil viviendas es de aproximadamente 240000 , pesos incluidos los gastos de formación del comité comunitario de salud, ejecución de planes de comunicación social y promoción de la salud, reuniones de seguimiento y evaluación del efecto de actividades.

Aunque no son percibidos durante la temporada de secas debido a que no acumulan agua, los contenedores de tamaño pequeño-mediano y de usos diversos toman mayor relevancia en la temporada de lluvias. Su eliminación o manejo, si se considera que la mayoría de estos utensilios son percibidos como de utilidad por la gente, deberá incluir la implementación de un programa educativo donde se contemple la recolección y destino final como desechos sólidos y / o su cuidado si se decide mantenerlos en el domicilio. Como parte de una política pública de la Secretaría del Medio Ambiente y Recursos Naturales, para la Prevención y Gestión Integral de los Residuos en México y como proyecto Residuos y Dengue, ${ }^{35,36}$ se realizó una propuesta para implementar una estrategia metodológica educativa llamada Reduce, Reutiliza y Recicla (RRR) en el Municipio de Xochitepec, Morelos, en la que se calculó el costo de 975325 pesos para capacitar a una población objetivo de 13414 habitantes; se consideró la implementación educativa, un vehículo recolector de desechos sólidos, separadores de residuos, materiales didácticos, construcción de compostas y la evaluación del empoderamiento de la estrategia. Esta propuesta metodológica podría ser inicialmente la más factible para el control de contenedores de tamaño pequeño-mediano, debido a que la población no los elimina durante las campañas de descacharrización. Los resultados presentados son consistentes con el concepto de intervenciones adaptativas para Ae. aegyp$t i{ }^{5,9} \mathrm{~A}$ lo largo de los periodos de muestreo en las tres localidades, se detectaron cambios considerables en la productividad de los contenedores clave. En la temporada de lluvias, los cuatro tipos de contenedores más importantes en términos de su productividad pupal, reportados para las tres localidades fueron, en orden de importancia, los diversos chicos; botes y cubetas; macetas y macetones, y trastes de cocina y lavado. Una estrategia de control enfocada hacia estos cuatro tipos de recipiente en las tres localidades durante las lluvias podría impactar a $63 \%$ de la población de adultos de Ae. aegypti. En la temporada de secas, la productividad pupal se restringe a criaderos particulares entre las localidades (macetas y macetones en Jojutla y Tlaquiltenango y tanques en Cuautla).

Las estrategias de control actuales podrían entonces estar dirigidas puntualmente hacia los tipos de criadero más productivos durante la temporada seca, y reforzar estas intervenciones en los meses previos a la temporada de lluvia. Actualmente, la campaña preventiva de promoción y comunicación social del estado de Morelos utiliza tres premisas; 1) Lava con jabón y cloro cubetas, piletas, tinacos o cisternas, 2) Tapa todo recipiente en el que almacenes agua y 3) Voltea o tira botellas, llantas, latas, macetas o cualquier objeto en el que se pueda acumular agua. Estos mensajes podrían tener mejor efecto al hacer énfasis en los criaderos más importantes (productivos) de acuerdo con los resultados encontrados, al considerar la temporalidad y la localidad, a través de medios de difusión locales (lonas, pinta de bardas, trípticos, folletos, perifoneo, radio, etc), como se describe en el cuadro III.

El riesgo entomológico conferido por los tanques/ pilas, las macetas y macetones, diversos chicos, botes y 
Cuadro III

Mensajes sugeridos para la población, de ACUERDo con los ReCIPIENTES PROductivos PARA CADA LOCALIDAD DE ESTUDIO

\begin{tabular}{|c|c|c|c|c|}
\hline Temporada & Mensaje a la población & $C$ & $J$ & $T$ \\
\hline \multirow{3}{*}{ Lluvias } & Voltea todos los botes, cubetas y recipientes grandes y pequeños cuando no sean usados & & $\mathrm{X}$ & $\mathrm{X}$ \\
\hline & Agujera las macetas y macetones para que tengan salida de agua & $\mathrm{X}$ & & \\
\hline & Limpia tu patio y tira llantas y recipientes pequeños inservibles & $\mathrm{X}$ & $\mathrm{X}$ & $\mathrm{X}$ \\
\hline \multirow{4}{*}{ Todo el año } & Lava todas las paredes internas de tambos y tanques y tápalos & $\mathrm{X}$ & $\mathrm{X}$ & $\mathrm{X}$ \\
\hline & Cría peces en tanques para combatir a los maromeros ${ }^{\ddagger}$ & $\mathrm{X}$ & $\mathrm{X}$ & $\mathrm{X}$ \\
\hline & Voltea botes, cubetas y recipientes grandes y pequeños cuando no sean usados & $\mathrm{X}$ & & \\
\hline & Agujera macetas y macetones para que tengan salida de agua & & $\mathrm{X}$ & $x$ \\
\hline
\end{tabular}

cubetas, y trastes de cocina y lavado resultó evidente en las tres localidades, por lo que se considera su persistencia y contribución a la productividad pupal en ambas temporadas. También demostró que el control de estos RPPU no se consigue durante las campañas actuales basadas en la descacharrización (por que la mayoría son considerados como útiles por los moradores) y, con excepción de los tanques/pilas, tampoco con la abatización, por lo que la participación de la comunidad en el control enfocado es a futuro la alternativa más costo rentable en términos de sustentabilidad.

La evidencia generada en Cuautla, Jojutla y Tlaquiltenango con respecto a los tipos de contenedores más productivos, indica que el o los criaderos productivos pueden variar geográficamente, por lo que las estrategias o campañas de prevención y control deberán basarse en información y evidencia de contextos locales. Estratificar la importancia relativa de los criaderos de acuerdo con su productividad en cada zona endémica o de alto riesgo de dengue podría resultar en un control de inmaduros y sus criaderos más efectivo y económico. Este trabajo representa el primer estudio de muestreo de productividad pupal de criaderos en el centro del país y demuestra la utilidad de los muestreos pupales en el contexto operativo para guiar y optimizar el control de los criaderos donde se está desarrollando el vector del dengue, aspecto que puede conducir a reducir el riesgo entomológico. No se contrapone a las estrategias actuales, y puede integrarse al plan operativo de los estados endémicos a dengue, y particularmente dentro de las áreas de elevado riesgo entomológico y de casos. Sin embargo, la eficacia de estas intervenciones en las densidades de mosquitos y finalmente, en la transmisión del dengue, tendrá que ser evaluada en futuros estudios en el estado de Morelos.

\section{Agradecimientos}

A los habitantes de Cuautla, Jojutla y Tlaquiltenango por su colaboración. A los SSM, por el apoyo proporcionado a este trabajo. Estos resultados son parte del proyecto "Estudio de la productividad de criaderos de Aedes aegypti en localidades de alto riesgo del estado de Morelos, México para la propuesta de una estrategia enfocada para el control de dengue" financiado por el FOSISS-2007.

Declaración de conflicto de intereses: Los autores declararon no tener conflicto de intereses.

\section{Referencias}

I. Secretaría de Salud (SSA). 200I. Programa de Acción: Prevención y Control de Enfermedades Transmitidas por Vector. México: SSA, 2001. 2. Secretaría de Salud. Programa de Acción específico 2007-2012, Dengue. México: SSA, 2008.

3. Focks DA.A Review of Entomological Sampling Methods and Indicators for Dengue Vectors. Documento TDR/IDE/Den/03. I. Geneva:World Health Organization, 2003.

4. Focks DA. Multicountry study of Aedes aegypti pupal productivity survey methodology: findings and recommendations. Documento TDR/IRM/ Den/06.I. Geneva:World Health Organization, 2006.

5. Koenraadt CJ,Aldstadt J, Kijchalao U, Sithiprasasna R, Getis A, Jones JW, et al. Spatial and temporal patterns in pupal and adult production of the dengue vector Aedes aegypti in Kamphaeng Phet, Thailand.Am J Trop Med Hyg 2008;79(2):230-238.

6. Barrera R,Amador M, Clark GG. Use of the pupal survey technique for measuring Aedes aegypti (Diptera: Culicidae) productivity in Puerto Rico. Am J Trop Med Hyg 2006;74(2):290-302.

7. Bisset JA, Marquetti MC, Suárez S, Rodríguez MM, Padmanabha H. 2006. Application of the pupal/demographic-survey methodology in an area of Havana, Cuba, with low densities of Aedes aegypti (L.).Ann Trop Med Parasitol 2006; 100: S45-S5I. 
8. Hammond SN, Gordon AL, Lugo EC, Moreno G, Kuan GM, López MM, et al. Characterization of Aedes aegypti (Diptera: Culicidae) production sites in Urban Nicaragua.J Medical Entomol 2007;44(5):85I-860. 9. Lenhart AE, Castillo CE, Oviedo M,Villegas E. Use of the pupal/ demographic-survey technique to identify the epidemiologically important types of containers producing Aedes aegypti (L.) in a dengue-endemic area of Venezuela. Ann Trop Med Parasitol 2006;100:S53-S59.

10. Morrison AC, Sihuincha M, Stancil JD, Zamora E, Astete H, Olson JG, et al. Aedes aegypti (Diptera: Culicidae) production from non-residential sites in the Amazonian city of lquitos, Peru. Ann Trop Med Parasitol 2006; 100:S73-S86.

II. Romero-Vivas CM, Arango-Padilla P, Falconar AK. 2006. Pupalproductivity surveys to identify the key container habitats of Aedes aegypt (L.) in Barranquilla, the principal seaport of Colombia. Ann Trop Med Parasitol 2006; 100:587-S95.

12. Troyo A, Calderón-Arguedas O, Fuller DO, Solano ME, Avendaño A, Arheart KL, et al. Seasonal profiles of Aedes aegypti (Diptera: Culicidae) larval habitats in an urban area of Costa Rica with a history of mosquito control.J Vector Ecol 2008;33(I):76-88.

13.Arredondo-Jiménez JI,Valdéz-Delgado KM. Aedes aegypti pupal/ demographic surveys in southern Mexico: consistency and practicality. Ann Trop Med Parasitol 2006; 100: SI7-S32.

14. Manrique-Saide P, Davies CR, Coleman PG, Rebollar-Tellez E, CheMendoza A, Dzul-Manzanilla F, et al. Pupal Surveys for Aedes aegypti Surveillance and Potential Targeted Control in Residential Areas of Mérida, México.AMCA 2008;24(2):289-298.

15. Servicios de Salud de Morelos. 2006. Programa Estatal del Programa Dengue. Morelos. México.

16. Secretaría de Salud. 2008. Plataforma Única de Dengue/Dirección General de Epidemiología. México, 2008.

17. García E. Modificaciones al sistema de clasificación climática de Koeppen. México: Universidad Nacional Autónoma de México, 1987:246. 18. Instituto Nacional de Ecología (INE). Informe Final. Análisis de la vulnerabilidad y capacidad de adaptación al cambio climático en los sectores más relevantes del estado de Morelos. México: Universidad Autónoma del Estado de Morelos, 2006:18I.

19. Pan American Health Organization (PAHO). Dengue and Dengue Hemorrhagic Fever in the Americas: Guidelines for prevention and control.Washington DC: 1994.

20. Darsie RF Jr. 195I. Pupae of culicine mosquitoes of the northeastern united status (Diptera, Culicidae, Culicini). New York: Cornell University Agricultural Experiment Station Ithaca, 1951: 67.

2I. Darsie RF,Ward RA. (198I). Identification and Geographical Distribution of the Mosquitoes of North America, North of México. Mosquito Systematics 198I;supp I: I-313.

22. Ibáñez-Bernal S, Martínez-Campos C. Clave para la identificación de larvas de mosquitos comunes en las áreas urbanas y suburbanas de la República mexicana. Fol Entomol Mexicana 1994;92:43-73.
23. Walter Reed Biosystematics Unit (WRBU). Keys to the medicaly important mosquito species. [Consultado 2008 agosto 15]. Disponible en: http://www.wrbu.org/northcom_MQkeys.html.

24. Focks DA, Chadee DD. Pupal survey: an epidemiologically significant surveillance method for Aedes aegypti: an example using data from Trinidad. Am J Trop Med Hyg 1997;56(2): 159- 167.

25. Morrison AC, Gray K, Getis A, Astete H, Sihuincha M, Focks D, et al. Temporal and geographic patterns for Aedes aegypti (Diptera: Culcidae) production in Iquitos, Peru. J Medical Entomol 2004;4| (6): I | 23- | | 42. 26. Focks DA, Brenner RJ, Hayes-Daniels J. 2000. Transmission threshold for dengue in terms of Aedes aegypti pupae per person with a discussion of their utility in source reduction efforts. Am J Trop Med Hyg 2000;62:1 I-18. 27. Morrison AC, Zielinski-Gutierrez E, Scott TW, Rosenberg R. Defining challenges and proposing solutions for control of the virus vector Aedes aegypti. PLoS Med 2008;5(3):e68.0362-0366.

28. Cifuentes E, Sánchez AM. Factores ambientales que determinan la aparición de brotes y la persistencia del dengue en Morelos. Salud Pública Méx 2007;49(sup. I):II4-II6.

29. Kroeger, Lenhart A, Ochoa M, Villegas E, Levy M, Alexander N, et al. Effective control of dengue vectors with curtains and water container covers treated with insecticide in Mexico and Venezuela: cluster randomised trials. BMJ 2006; 332:1247-1252.

30. Caballero-Hoyos R, Torres-López T, Chong-Villarreal F, Pineda-Lucatero A,Altuzar-González M, López-Coutiño B. Cultural conceptions on dengue in urban contexts in Mexico. Rev Saude Publica 2006;40(I):126-133.

3I. Danis-Lozano R, Rodríguez MH, Hernández-Ávila M. Gender-related family head schooling and Aedes aegypti larval breeding risk in southern Mexico. Salud Publica Mex 2002;44(3):237-242.

32. Barrera R,Amador M, Clark G. Ecological Factors Influencing Aedes aegypti (Diptera: Culicidae) Productivity in Artificial Containers in Salinas, Puerto Rico.J Med Entomol 2006;43(3):484-492.

33. Vezzani D,Albicócco $A$. The effect of shade on the container index and pupal productivity of the mosquitoes Aedes aegypti and Culex pipiens breeding in artificial containers. Medical and Veterinary Entomology 2009;23: 78-84

34. Coe G, Llanos M. Manual de comunicación social para programas de promoción de la salud de los adolescentes. Washington, DC: Organización Panamericana de la Salud, 200I:15. [Consultado]. Disponible en: http:/l www.paho.org/Spanish/HPP/HPF/ADOL/ComSocial.pdf.

35. Secretaría de Medio Ambiente y Recursos Naturales. Programa Nacional para la Prevención y Gestión Integral de los Residuos Sólidos. [Consultado 2009 abril 3]. Disponible en: http://www.semarnat.gob.mx/ programas subsidios/Documents/PNPGIR.pdf.

36. Cortinas C. Guía práctica para desarrollar planes de manejo de residuos sólidos en las escuelas para su reducción, reutilización o reciclado (3R) Documento en línea. [Consultado 2009 abril I]. Disponible en: http://www.cristinacortinas.net/images/residuos/residuosydengue/l4_ manejoescuelas.pdf. 